\title{
A new two-step accurate CT-MRI fusion technique for post-implant prostate cancer
}

\author{
Hiroaki Kunogi, MD, PhD!, Hidehiro Hojo, MD, PhD!, Yoshiaki Wakumoto, MD, PhD², Anneyuko I. Saito, MD, PhD!, \\ Satoshi Ishikura, MD, PhD', Yuki Yamashiro, MD³, Prof. Ryouhei Kuwatsuru, MD, PhD³. Prof. Keisuke Sasai, MD, PhD' \\ 'Department of Radiation Oncology, ${ }^{2}$ Department of Urology, ${ }^{3}$ Department of Radiology, Juntendo University. Tokyo, Japan
}

\begin{abstract}
Purpose: To develop an accurate method of fusing computed tomography $(\mathrm{CT})$ with magnetic resonance imaging (MRI) for post-implant dosimetry after prostate seed implant brachytherapy.

Material and methods: Prostate cancer patients were scheduled to undergo CT and MRI after brachytherapy. We obtained the three MRI sequences on fat-suppressed T1-weighted imaging (FST1-WI), T2-weighted imaging (T2-WI), and $2^{*}$-weighted imaging (T2*-WI) in each patient. We compared the lengths and widths of 450 seed source images in the 10 study patients on CT, FST1-WI, T2-WI, and T2*-WI. After CT-MRI fusion using source positions by the leastsquares method, we decided the center of each seed source and measured the distance of these centers between CT and MRI to estimate the fusion accuracy.

Results: The measured length and width of the seeds were $6.1 \pm 0.5 \mathrm{~mm}$ (mean \pm standard deviation) and $3.2 \pm 0.2 \mathrm{~mm}$ on CT, $5.9 \pm 0.4 \mathrm{~mm}$, and $2.4 \pm 0.2 \mathrm{~mm}$ on FST1-WI, $5.5 \pm 0.5 \mathrm{~mm}$ and $1.8 \pm 0.2 \mathrm{~mm}$ on T2-WI, and $7.8 \pm 1.0 \mathrm{~mm}$ and 4.1 $\pm 0.7 \mathrm{~mm}$ on T2*-WI, respectively. The measured source location shifts on CT/FST1-WI and CT/T2-WI after image fusion in the 10 study patients were $0.9 \pm 0.4 \mathrm{~mm}$ and $1.4 \pm 0.2 \mathrm{~mm}$, respectively. The shift on CT/FST1-WI was less than on CT/T2-WI $(p=0.005)$.

Conclusions: For post-implant dosimetry after prostate seed implant brachytherapy, more accurate fusion of CT and T2-WI is achieved if CT and FST1-WI are fused first using the least-squares method and the center position of each source, followed by fusion of the FST1-WI and T2-WI images. This method is more accurate than direct image fusion.
\end{abstract}

Key words: brachytherapy, fusion, magnetic resonance imaging, post-implant, prostate cancer.

\section{Purpose}

Permanent prostate brachytherapy with ${ }^{125} \mathrm{I}$ is a standard treatment option for patients with low- or intermediate-risk prostate cancer [1,2]. Post-implant dosimetry (PID) for brachytherapy is important to evaluate prostate implant quality [3] and is mandatory for all patients undergoing these treatments [4]. Computed tomography (CT) is the standard imaging modality for PID, and it is easier to identify all implanted seeds by CT scan than by magnetic resonance imaging (MRI). However, delineating pelvic organs on a CT scan is difficult. The contouring of the prostate is more reproducible on T2-weighted MR images than CT or transrectal ultrasound images [5-8]. Without the precise post-implant registration of CT and MRI data sets, the quality of PID is not satisfactory [9-13]. This study investigated a potential MR sequence for the accurate fusion of $\mathrm{CT}$ and MR images for PID. The study was approved by the Ethics Board of our institution.

\section{Material and methods \\ Permanent prostate brachytherapy}

Between January and October of 2012, 10 low-risk (T1-2a, PSA < $10 \mathrm{ng} / \mathrm{ml}$ and Gleason score $\leq 6)$ prostate cancer patients underwent transperineal interstitial permanent prostatic implantation of loose ${ }^{125} \mathrm{I}$ radioactive seeds (4.55 × $0.97 \mathrm{~mm}$; Oncoseed 6711, GE Healthcare MediPhysics Inc., Arlington Heights, IL, USA) at our institution. A total of 636 seed sources were implanted in the 10 study patients. The sources were peripherally loaded using a Mick applicator (Mick Radio-Nuclear Instruments Inc., Mount Vernon, NY, USA), and the activity per seed was $0.338 \mathrm{mCi}$. Variseed ver. 8.0 (Varian Medical Systems 
Inc., Palo Alto, CA, USA) software was used for PID. Neoadjuvant hormonal therapy was performed to adjust the prostate volume from 15 to $30 \mathrm{ml}$.

\section{Post-implant image acquisition}

Four weeks after prostate brachytherapy, CT and MRI data were acquired for dosimetric analysis according to the recommended schedule [14]. Three imaging sequences were obtained: fat-suppressed T1-weighted imaging (FST1-WI), T2-weighted imaging (T2-WI), and $\mathrm{T} 2{ }^{*}$-weighted imaging $\left(\mathrm{T} 2^{*}-\mathrm{WI}\right)$ using a Siemens Avanto 1.5T MRI scanner (Siemens, Munich, Germany) with $3 \mathrm{~mm}$-thick-slices. The technical parameters of FST1-WI were as follows: repetition time (TR)/echo time (TE) in ms, 955/150; in-plane resolution, $1.0 \times 0.7 \mathrm{~mm}^{2}$. The technical parameters of T2-WI were: TR/TE, 5000/100; in-plane resolution, $1.0 \times 0.7 \mathrm{~mm}^{2}$; Turbo Spin Echo factor, 15 . The technical parameters of $\mathrm{T} 2 *$-WI were: TR/TE, 600/19; in-plane resolution, $1.1 \times 0.7 \mathrm{~mm}^{2}$. FST1-WI, T2-WI, and T2*-WI were obtained successively. During PID, the prostate is contoured using T2-WI to estimate prostate volume.

Within $1 \mathrm{~h}$ of MRI, a post-implant CT was performed using a GE spiral CT (Hi-Speed Dxi; GE Healthcare, Buckinghamshire, UK). During the CT scan, a urinary catheter (8 Fr) was inserted. Sequences of $2 \mathrm{~mm}$ slices were acquired using a $50 \mathrm{~mm}$ field of view (FOV), and a pitch of $2 \mathrm{~mm} / 2 \mathrm{~mm}=1$ (defined as the table feed/total detector width of the collimated beam). No intravenous contrast material was used during the CT or MRI scans.
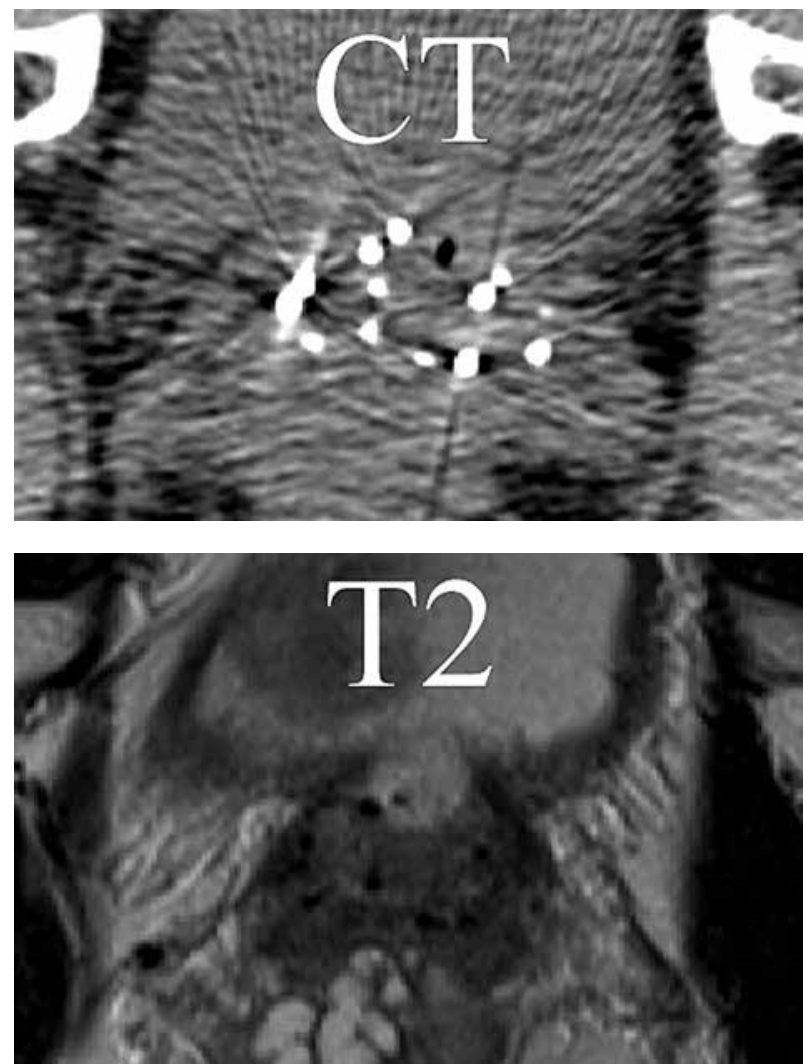

\section{Comparison of source images in CT, FST1-WI,} T2-WI, and T2*-WI

We measured the lengths and widths of the source images on CT, FST1-WI, T2-WI, and T2*-WI, and compared the data among the modalities (Fig. 1). We determined the degrees of signal intensity loss on MRI to be those of the seeds. Overlapping source images and extra-prostatic areas that might have increased the inaccuracy of size measurements were excluded from the analysis. Seeds with distinct outlines in an intra-prostatic area were selected for this study. Of the 636 seed sources, 186 were found to be overlapped and located in an extra-prostatic area; these 186 sources were excluded from the study. Consequently, 450 seed sources were measured.

\section{Fusion method}

We accomplished the registration using 12 geometric centers of the seed distribution by a least-squares method [15] between CT and each MRI sequence (FST1-WI and T2-WI) using the Variseed 8.0 software. The two distinct outline seeds were selected on either side (right and left lobe) at the base, middle, and apex of the prostate ( 12 seed centers total), and the 12 source centers were used for seed match registration. Extra-prostatic sources were not used. The width of the $\mathrm{T} 2{ }^{*}$-WI source images appeared nearly twice as long as the seed clarifying sequences (FST1-WI). Because of the considerable loss of $\mathrm{T} 2 *-\mathrm{WI}$ signal intensity, many of the sources overlapped. Detection of the seed centers on $\mathrm{T} 2 *$-WI was difficult enough that an estimation
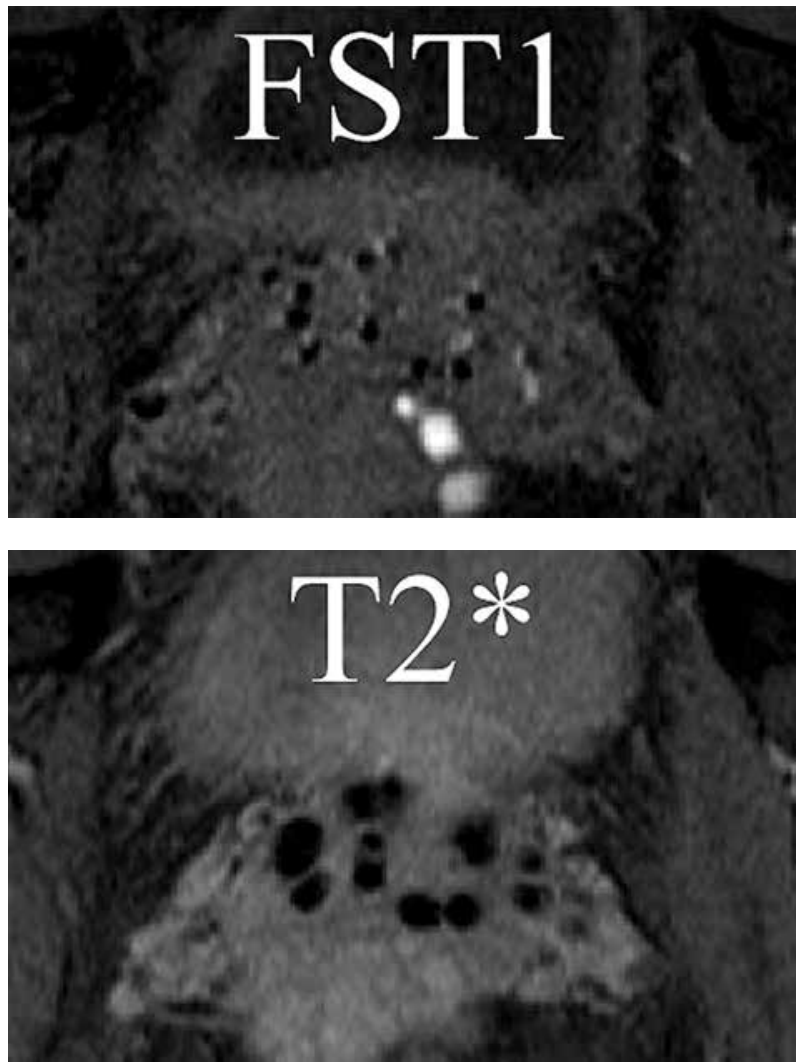

Fig. 1. CT, FST1-WI, T2-WI, and T2*-WI of the pelvis with seed sources 
of the fusion accuracy between CT images and $\mathrm{T} 22^{*}-\mathrm{WI}$ was not performed.

\section{Estimation of the fusion accuracy}

In each patient, the source location shifts on CT/FST1WI and CT/T2-WI were calculated from source location data using the VariSeed Source Location Export function in Variseed 8.0. The mean shifts for all sources were calculated and estimated for each patient.

\section{Statistics}

To assess the fusion accuracy between CT and each MRI sequence, each data point was compared using the paired-sample $t$ test. Analyses were performed using SPSS, version 18 (SPSS Inc., Chicago, IL, USA). $P$ values $<0.05$ were considered statistically significant.

\section{Results}

The estimated mean prostate volume was $21.6 \mathrm{ml}$ (range 13.1-32.8 ml). The median number of intra-prostatic seeds per patient was 45 (range 29-59, with a total of 450 sources). The measured lengths and widths of the seeds were $6.1 \pm 0.5 \mathrm{~mm}$ [mean \pm standard deviation], and $3.2 \pm 0.2 \mathrm{~mm}$ on CT, $5.9 \pm 0.4 \mathrm{~mm}$ and $2.4 \pm 0.2 \mathrm{~mm}$ on FST1-WI, $5.5 \pm 0.5 \mathrm{~mm}$ and $1.8 \pm 0.2 \mathrm{~mm}$ on T2-WI, and 7.8 $\pm 1.0 \mathrm{~mm}$ and $4.1 \pm 0.7 \mathrm{~mm}$ on T2*-WI, respectively (Fig. 2 ). The measured source location shifts on CT/FST1-WI and CT/T2-WI after image fusion in the 10 study patients were $0.9 \pm 0.4 \mathrm{~mm}$ and $1.4 \pm 0.2 \mathrm{~mm}$, respectively. The shift on CT/FST1-WI was less than on CT/T2-WI $(p=0.005)$.

\section{Discussion}

This study compared the accuracy of seed match registration using the least squares method with different MRI sequences and concluded that the sequences that more precisely define the seeds resulted in a better match. Additional MRI sequences were then matched to this registration, under the assumption that they would match since these images were obtained at the same time from the same position. The present study determined that FST1-WI was the most suitable MRI sequence for $\mathrm{CT} / \mathrm{MRI}$ fusion. The differences in the size of the source images between CT and FST1-WI were the smallest; thus, we accomplished the registration easily and accurately by using the geometric centers of the source images on each image by the least-squares method. We invented a new CT-MRI fusion technique using FST1-WI-namely, a two-step image-fusion method using two sets of images (CT and FST1-WI, and FST1-WI and T2-WI). In the first step, fusion of CT images and FST1-WI was performed by matching selected seed positions on each image. The least squares method was used during the first step, because McLaughlin et al. previously reported that registration could be accurately accomplished using seed positions by this method [15]. In the next step, both CT and FST1-WI obtained from the first step could be accurately matched to T2-WI, because FST1-WI and T2-WI were co-registered. This two-step image fusion technique pro-

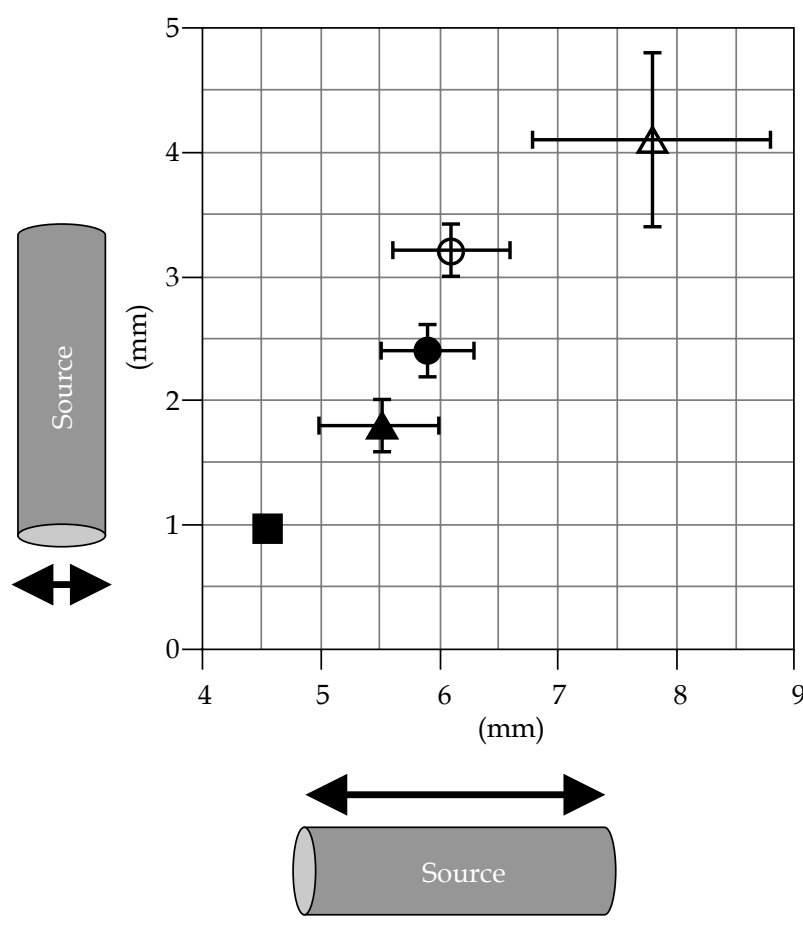

O $\mathrm{CT} \quad \mathrm{FST} 1_{\mathrm{T} 2} \quad \Delta \mathrm{T} 2^{*} \quad$ Actual seed $^{*}$

CT - computed tomography, FST1-WI - fat-suppressed T1-weighted imaging, T2-WI-T2-weighted imaging, $T 2^{*}-W I-T 2^{*}$-weighted imaging, MRI - magnetic resonance imaging

Fig. 2. Measured lengths and widths of the seeds on CT, FST1-WI, T2-WI, and T2*-WI. The data show the actual length (horizontal axis) and width (vertical axis) for reference $(4.55 \times 0.97 \mathrm{~mm})$, and measured lengths (horizontal axis) and widths (vertical axis) of the seeds on CT, FST1-WI, $\mathrm{T} 2-\mathrm{WI}$, and T2*-WI. The error bars represent one standard deviation from the means of the 450 seed sources

vides high-quality CT-MRI fusion images. Also, in a clinical setting, the accuracy of the image fusion obtained from the two-step fusion technique can be evaluated by eye as the shift in seed between the fused CT images and FST1-WI. This is a critically important method for evaluating the fusion accuracy.

Computed tomography and T2-WI were fused more accurately using the two-step image-fusion technique than using direct image fusion. We compared the twostep image-fusion and direct image-fusion measuring source location shifts on CT/FST1-WI and CT/T2-WI after the seed match registration using 12 geometric seed centers by the least-squares method. The shift was significantly less on CT/FST1-WI than on CT/T2-WI $(p=0.005)$.

The two-step fusion method is an approximation, and a residual mismatch may require an anatomic match and correction. We acknowledge that errors may occur with the least squares method and that one sequence may merely lead to a better match than another. At the mid-prostate, the contour of the prostate is relatively clear on CT. After seed to seed registration, CT images of the mid-prostate may reveal that seed to seed registration by the least squares method is in fact only an approximation and not always adequate. This overlay of mid-prostate 
images will enable identification of residual mismatches that require an anatomic match and correction.

Katayama et al. demonstrated that T2*-WI is useful for the detection of seeds without intravenous contrast media [16]. However, metallic implants such as seed sources cause considerable loss of signal intensity in $\mathrm{T} 2^{*}-\mathrm{WI}$ (Fig. 1) [17]. Detection of the center of implanted seeds by $\mathrm{T} 2^{*}$-WI is difficult.

Several other image fusion methods can be used, such as fusion by bony anatomy, mutual information (MI) registration, and visual manual seed match registration, using available software. Fusion by bony anatomy is suboptimal in terms of accuracy and reproducibility. Roberson et al. showed that MI registration using large-image data sets of the same anatomic volume was more substantial than seed match $\mathrm{CT} / \mathrm{T} 2 \mathrm{MR}$ registration, and approximately $30 \mathrm{~min}$ was needed for MI registration compared with several hours for seed match registration [18]. However, given the independent motion of the prostate compared with pelvic soft tissue, MI registration using anatomic image data sets will be inaccurate. Our two-step intra-prostatic seed match MR registration can be achieved as quickly as and is more accurate than MI registration for the prostate. Visual manual seed to seed registration on T2-weighted MRI to CT images using available software is commonly performed; however, this method is more time consuming than our two-step method. As the next step of research, it would be interesting to quantitatively compare our method to mutual information or simply landmark registration.

Maletz et al. reported a method [9] requiring the use of specialized MIMVista software. Conversely, in the present study, image fusion and evaluation were performed using the more common Variseed software. To evaluate image fusion quality, Maletz et al. compared dose volume parameters for the target volume. However, the parameters they used were dependent upon prostate contouring and the dose distribution after implantation. The evaluation of dose volume parameters is not a substantiated method for measuring fusion accuracy. In the present study, we examined the distance of the same seed between fused CT and MR images to evaluate the fusion accuracy. Since it is not dependent on dose volume parameters, the evaluation methodology is preferred.

In the present study, seed match registration was performed using the least squares method with 12 geometric centers between CT images and each MRI sequence (FST1-WI and T2-WI). Registration using 12-seed centers may be a practical first step; without a systematic comparison of seed numbers, we cannot conclude that 12 is an adequate number. Roberson et al. attempted to define all seeds on T2-WI during seed match registration [18] However, it is difficult to visualize all implanted seed sources, and the inclusion of vague sources may decrease fusion accuracy.

It is possible to estimate PID using MRI alone. However, visualizing all implanted seeds on MRI, even on FST1-WI, is difficult. Detecting seed source positions is easier using CT, particularly using the seed-finder facility in Variseed 8.0. Thus, the CT-MRI fusion method was advocated [4,19].
In this study, the post-implant prostate volume was not large (mean, $21.6 \mathrm{ml}$; range, 13.1-32.8 ml) because of neoadjuvant hormonal therapy to adjust the prostate volume. In cases involving a large prostate, the activity per seed in accordance with prostate volume will be adequately selected. Our two-step image-fusion technique does not rely heavily on prostate volume; therefore, our results may be extrapolated to larger prostates. In this study, of the 636 seed sources, $186(29 \%)$ were found to be overlapping and located in an extra-prostatic area. In cases that involve larger prostates with stronger activity per seed, fewer overlapping source images may be found than in this study.

One limitation of the present study was the assumption that since the images were acquired at the same time, the optimally matched sequence can be matched to other MRI sequences. Magnetic resonance imaging scans take several minutes to perform, and multiple sequences require at least 15 minutes of acquisition time, during which the bowel and bladder may displace the prostate from the initial scan position.

Another fundamental limitation of the present study was that the seed position was displaced by slice imaging (CT or MRI) in proportion to the slice thickness. In fact, when seed length and width variation were plotted against MRI sequence (Fig. 2), all imaging methods including CT led to an overestimation of the length and width compared with the actual parameters $(4.55 \times 0.97 \mathrm{~mm})$. Due to the shift of every seed by CT and MRI, the seed center was shifted as well. With seed length consistently longer than the slice thickness, it creates uncertainty regarding the actual seed position. This uncertainty is unavoidable as long as seed position is obtained using slice imaging in proportion to the slice thickness.

Another limitation was the potential variation in the appearance of different source models, and these differences may affect the fusion accuracy of the two-step fusion technique. However, the ability of FST1-WI to more precisely define the sources can result in a better seed match. Additional measurements using different source models will need to be obtained in the future.

\section{Conclusions}

Computed tomography and T2-WI were fused more accurately using the two-step image-fusion technique than using direct image fusion. The two-step technique was performed by first fusing CT and FST1-WI by the least-squares method using each source center position, followed by fusion of the first-step image with a T2-WI.

\section{Disclosure}

Authors report no conflict of interest.

\section{References}

1. Ragde H, Blasko JC, Grimm PD et al. Interstitial iodine-125 radiation without adjuvant therapy in the treatment of clinically localized prostate carcinoma. Cancer 1997; 80: 442-453.

2. Wallner K, Roy J, Harrison L. Tumor control and morbidity following transperineal iodine 125 implantation for stage T1/T2 prostatic carcinoma. J Clin Oncol 1996; 14: 449-453. 
3. Moerland MA, Wijrdeman HK, Beersma R et al. Evaluation of permanent I-125 prostate implants using radiography and magnetic resonance imaging. Int J Radiat Oncol Biol Phys 1997; 37: 927-933.

4. Tanaka O, Hayashi S, Matsuo M et al. Comparison of MRI based and CT MRI fusion based postimplant dosimetric analysis of prostate brachytherapy. Int J Radiat Oncol Biol Phys 2006; 66: 597-602.

5. Smith WL, Lewis C, Bauman G et al. Prostate volume contouring: A 3D analysis of segmentation using 3DTRUS, CT, and MR. Int J Radiat Oncol Biol Phys 2007; 67: 1238-1247.

6. Lee WR, Roach M 3rd, Michalski J et al. Interobserver variability leads to significant differences in quantifiers of prostate implant adequacy. Int J Radiat Oncol Biol Phys 2002; 54: 457-461.

7. Roach M 3rd, Faillace-Akazawa P, Malfatti $C$ et al. Prostate volumes defined by magnetic resonance imaging and computerized tomographic scans for three dimensional conformal radiotherapy. Int J Radiat Oncol Biol Phys 1996; 35: 10111018.

8. Gomez-Iturriaga A, Crook J, Casquero F et al. Impact of intraoperative MRI/TRUS fusion on dosimetric parameters in cT3a prostate cancer patients treated with high-dose-rate real-time brachytherapy. J Contemp Brachytherapy 2014; 6: 154-160.

9. Maletz KL, Ennis RD, Ostenson J et al. Comparison of CT and MR-CT fusion for prostate post-implant dosimetry. Int J Radiat Oncol Biol Phys 2012; 82: 1912-1917.

10. Polo A, Cattani F, Vavassori A et al. MR and CT image fusion for postimplant analysis in permenent prostate seed implants. Int J Radiat Oncol Biol Phys 2004; 60: 1572-1579.

11. Vidakovic S, Jans HS, Alexander A, Sloboda RS. Post-implant computed tomography-magnetic resonance prostate image registration using feature line parallelization and normalized mutual information. J Appl Clin Med Phys 2006; 8: 21-32.

12. Tanaka O, Hayashi S, Sakurai K et al. Importance of the CTMRI fusion method as a learning tool for CT-based postimplant dosimetry in prostate brachytherapy. Radiother Oncol 2006; 81: 303-308.

13. Amdur RJ, Gladstone D, Leopold KA, Harris RD. Prostate seed implant quality assessment using MR and CT image fusion. Int J Radiat Oncol Biol Phys 1999; 43: 67-72.

14. Prestidge BR, Bice WS, Kiefer EJ, Prete JJ. Timing of computed tomography-based postimplant assessment following permanent transperineal prostate brachytherapy. Int J Radiat Oncol Biol Phys 1998; 40: 1111-1115.

15. McLaughlin PW, Narayana V, Drake DG et al. Comparison of MRI pulse sequences in defining prostate volume after permanent implantation. Int J Radiat Oncol Biol Phys 2002; 54: 703-711.

16. Katayama N, Takemoto M, Yoshio $\mathrm{K}$ et al. $\mathrm{T} 2^{*}$-weighted image/T2-weighted image fusion in postimplant dosimetry of prostate brachytherapy. J Radiat Res 2011; 52: 680-684.

17. Chavhan GB, Babyn PS, Thomas B et al. Principles, techniques, and applications of $\mathrm{T} 22^{*}$-based MR imaging and its special applications. Radiographics 2009; 29: 1433-1449.

18. Roberson PL, McLaughlin PW, Narayana V et al. Use and uncertainties of mutual information for computed tomography / magnetic resonance (CT/MR) registration post-permanent implant of the prostate. Med Phys 2005; 32: 473-482.

19. Bloch BN, Lenkinski RE, Helbich TH et al. Prostate postbrachytherapy seed distribution: comparison of high-resolution, contrast-enhanced, T1- and T2-weighted endorectal magnetic resonance imaging versus computed tomography: initial experience. Int J Radiat Oncol Biol Phys 2007; 69: 70-78. 